\title{
Formation and Disproportionation of Arene Sulfenic Acids
}

\author{
Sujit Ranjan Acharjee ${ }^{1}$ and S K Bhattacharjee ${ }^{2}$ \\ ${ }^{1}$ Department of Chemistry, Hojai College, Hojai-782435, Assam, India. \\ ${ }^{2}$ Department of Chemistry, Gauhati University, Guwahati-781014, Assam, India.
}

\section{Introduction}

Sulfenic acids, R-SOH, may be regarded as derivative of unknown inorganic acid, $\mathrm{H}_{2} \mathrm{SO}_{2}$ that may be derived from highly unstable oxide of sulfur, sulfur monoxide, SO. RSOH may be obtained by hydrolytic cleavage of S-X bond in sulfenyl halides, RSX. Sulfenic acids exist as RS-OH rather than

$$
R_{-}=0
$$

due to the fact that a formal $\mathrm{S}=\mathrm{O}$ bond energy $(373 \mathrm{KJ} / \mathrm{mol})$ is not much higher than the $\mathrm{S}-\mathrm{O}$ bond energy $(340$ $\mathrm{KJ} / \mathrm{mol}$ ) so that the loss of the strong $\mathrm{O}-\mathrm{H}$ bond energy would not be compensated for in the $\mathrm{S}=\mathrm{O}$ bond.

In many respects these oxyacids of sulfur are rather esoteric compounds. Their instability arises from their ability of forming intermolecular hydrogen bonding which results in self-condensation to thiolsulfinate esters (I)

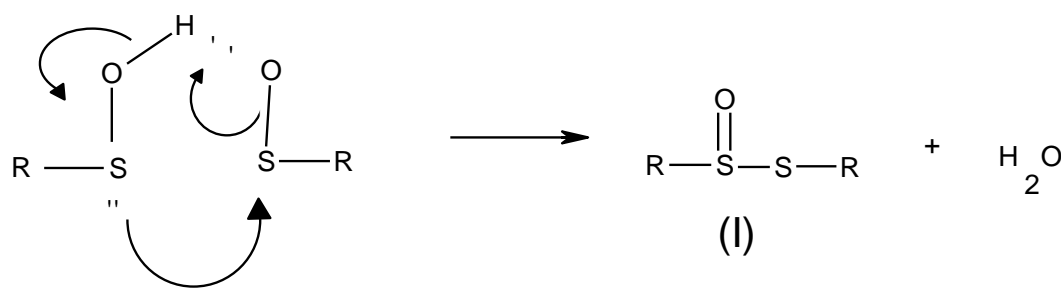

The fate of thiolsulfinate esters (I) depends on the $\mathrm{pH}$ of the medium.

At $\mathrm{pH} \leq 7$ the thiolsulfinate esters undergo disproportionation reaction to form corresponding disulfides (II) and thiolsulfonates (III)<smiles>[R]SS([R])=O</smiles>

(II)<smiles>[R]SS([R])(=O)=O</smiles>

(III)

At $\mathrm{pH}>8$ thiolsulfinates (I) form salts of sulfinicacids (IV) and disulfides (II)

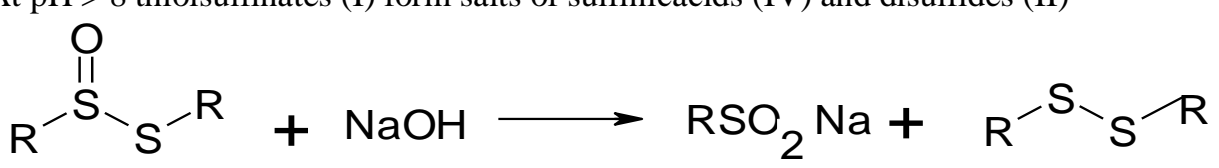

For these reasons only a very few stabilized sulfenic acids could be prepared till today. The aim of the present work is to prepare a stabilized aromatic sulfenic acid.

\section{Results \& Discussion}

4'-Nitroazobenzene-2-sulfenyl bromide (v) has been synthesized as a stabilized sulfenium carrier for the purpose. Since the inherent instability of sulfenic acids lies primarily in the ability of the S-OH group to undergo intermolecular hydrogen bonding and also on the nucleophilicity of the sulfur atom increase in the bulk of the Ar group in $\mathrm{Ar}-\mathrm{SOH} \&$ in the presence of an electron withdrawing group in the Ar moiety should prevent the self condensation of SOH group to thiolsulfinate esters (I). In the compound (V) the 2-(4'-Nitro) phenylazophenyl unit is a bulky group \& the azo group is electron withdrawing. Moreover the azo group acts as 
a good proton acceptor from the sulfenic acid unit to form a stabilized tautomeric structure (VII) of the acid (VI).<smiles>C#CC#C</smiles>

(VI)<smiles>O=[N+]([O-])c1ccc(-n2[nH]c3ccccc3so2)cc1</smiles>

(VII)

So, it is expected that the sulfenyl bromide (V), on hydrolytic cleavage of the S-Br bond should result in a stabilized sulfenic acid (VI, VII)<smiles>O=[N+]([O-])c1ccc(/N=N/c2ccccc2SBr)cc1</smiles>

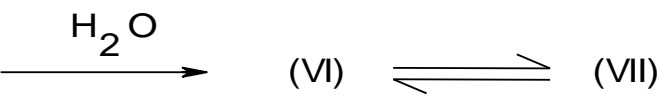

(V)

In an attempt to prepare the sulfenic acid (VI) an aqueous solution of the sulfenyl bromide (V) is treated with equimolecular amount of aq. $\mathrm{NaOH}$. The solution remains clear for half a minute \& then begins to turn turbid \& in about two hours time an orange yellow ppt is formed which is composed of a mixture of three compounds viz. the corresponding disulfide (VIII), thioether (IX), and a sulfenyl sulfite (X). Formation of these compounds can be explained due to disproportionation reaction of sulfenic acid (VI) initially formed involving a sulfenic anhydride (XI) intermediate. The unstable sulfenic acid self condenses to the thiolsulfinate (XI), which partly rearranges to the sulfenic anhydride (XII) giving the disproportionation products, the disulfide (VII) and the thiolsulfonate (XIII).

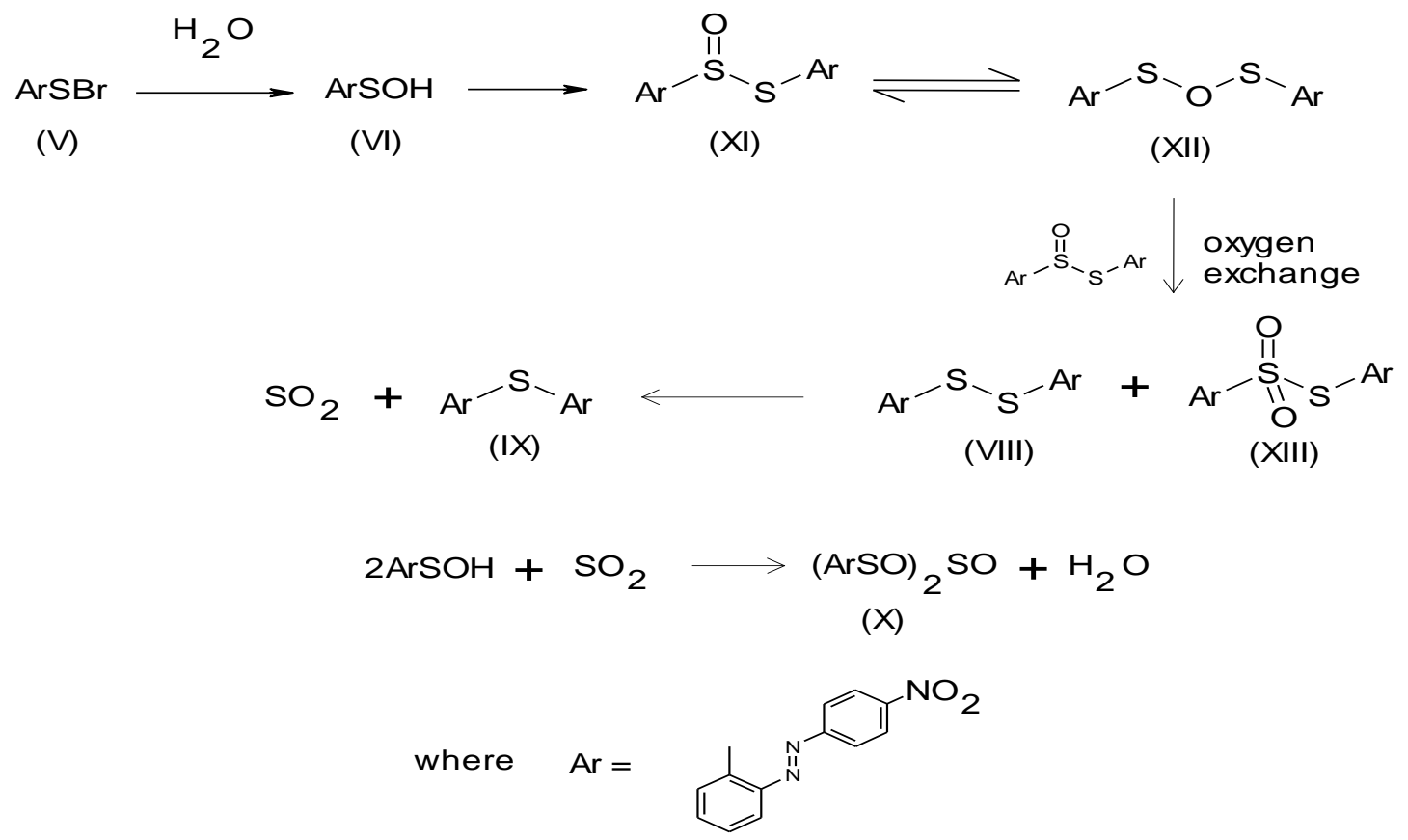

(XIII) Being unstable, splits off a molecule of SO2 to be converted to the diarylthioether (IX). The liberated $\mathrm{SO} 2$ in aq. solution, reacts with unconverted sulfenic acid still left to form the 'sulfinate' (X). The thermolability 
of the thiolsulfonate (XIII) has also been established by a direct synthesis of the compound from the corresponding sodium sulfinate (XIV) \& sulfenyl bromide (V) when a yellow flocculant ppt is obtained from the from the aq. solution.

This yellow ppt slowly turns red during filtration \& crystallization to give the diarylthioether (IX) \& SO2 gas which turns yellow dichromate paper green.

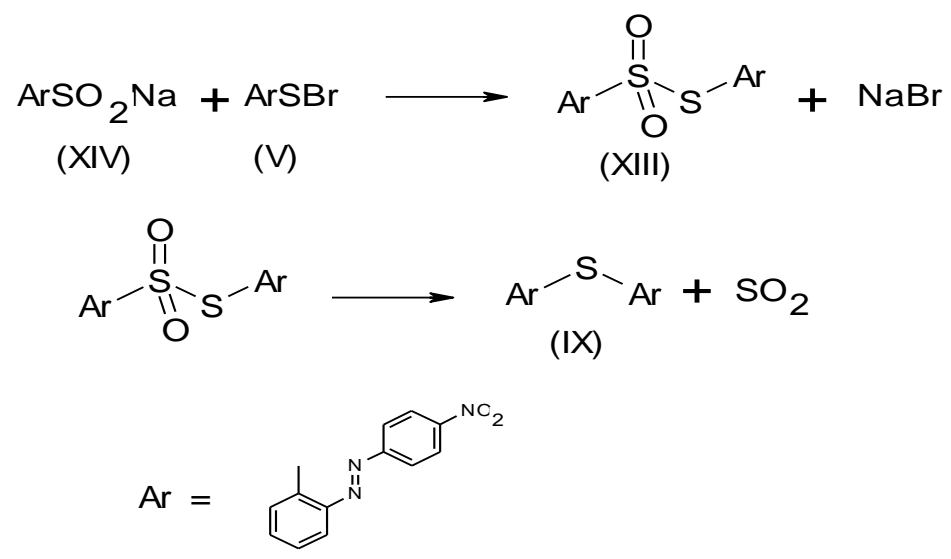

When the hydrolysis has been performed three molar proportion of alkali, the reaction takes place much faster due to the formation of sulfenate salt (XV) which imparts a transient blue violet colour to the solution due to charge transfer phenomenon in the sulfenate anion. The sulfenic anion being more nucleophilic than the sulfenic acid undergoes self-condensation to thiolsulfinate (XI) much faster. The electrophilic sulfenyl sulfur of (XI) is readily attacked by excess alkali to form the corresponding soluble sodium sulfinate (XVI) and the mercaptan (XVII). The latter undergoes a rapid condensation with any unreacted sulfenic acid to form the corresponding insoluble disulfide (VIII). The formation of the products can be explained as per the scheme II.

Scheme- II
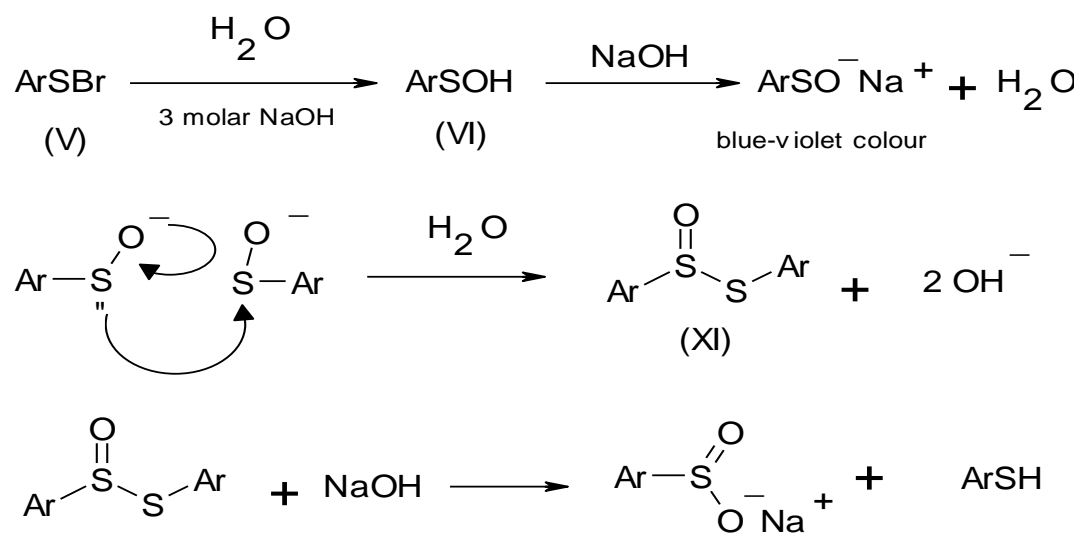

$(\mathrm{XVl})$

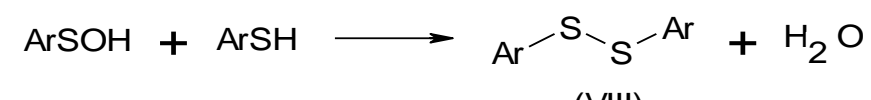

(VIII)

The free sulfinic acid (XIX) can be liberated as a rose red ppt by acidification of the aq. filtrate containing the sodium sulfinate. On crystallization from benzene-light petrol mixture this rose red sulfinic acid (XIX) gives colourless plates of the tautomeric form (XX) of the sulfenic acid. 
<smiles>O=S(=O)(O)O[Nb]</smiles>

$(\mathrm{XVI})$<smiles>O=[N+]([O-])c1ccc(N=Nc2ccccc2S(=O)(=O)O)cc1</smiles>

$(\mathrm{XIX})$<smiles>O=[N+]([O-])c1ccc(N2Nc3ccccc3S(=O)O2)cc1</smiles>

$(X X)$

Our endeavor to prepare a stabilized sulfenic acid has failed perhaps, due to the fact that the conjugate acid of $\mathrm{OH}$ being a very weak acid $\mathrm{H} 2 \mathrm{O}$, the sulfenyl sulfur atom of the sulfenic acid group S-OH remains strongly nucliophilic. This keeps the -SOH group away from the ortho arylazo group thereby precluding any proton transfer from $-\mathrm{SOH}$ to the azo group $-\mathrm{N}=\mathrm{N}$-, to attain tautomeric stability. Though the bulky ortho arylazo naphthyl moiety attached with the sulfur atom raises the activation energy of the self condensation reaction to the thiolsulfinate, as is reflected in the relatively long time ( 2 hour) taken for the completion of the reaction with equimolar amount of $\mathrm{NaOH}$, it can not prevent the reaction but only can slow it down.

\section{Experimental \\ REACTION WITH EQUIMOLAR SODIUM HYDROXIDE}

To a solution of the sulfenyl bromide $(1.068 \mathrm{gm}, \sim 0.003 \mathrm{M})$ in water $(100 \mathrm{ml})$ was added a solution of sodium hydroxide $(5.8 \mathrm{ml}$ of $2 \%$ solution, $\sim 0.003 \mathrm{M})$. The mixture was stirred well. The clear solution started becoming turbid in about 30 second $\&$ precipitation of a yellow substance was completed in about 2 hours. It was filtered off, washed \& dried.

Yield: $0.72 \mathrm{gm}$

This dried solid was now boiled with benzene $(20 \mathrm{ml})$. The sulfite $(\mathrm{X})$ remained insoluble \& was filtered off and was washed with a little benzene and dried.

Yield: $90 \mathrm{mg}$

Crystallized from rectified spirit as orange yellow needles. Mp: 192-93

\section{Analysis: C24H16N6O7S3}

Requires: C, 43.5; H, $2.4 \& \mathrm{~N}, 12.6 \mathrm{pc}$

Found: C, 43.8; H, $2.1 \& \mathrm{~N}, 12.9 \mathrm{pc}$

\section{UV \& visible spectra:}

$\lambda \max \left(\mathrm{nm}\right.$, ethanol): $422(\varepsilon \dot{m a x} 1500 ; \mathrm{n} \rightarrow \pi *)$, 352(Emax 12000; $\left.\pi \rightarrow \pi^{*}\right)$

\section{IR spectra:}

$v(/ \mathrm{cm}, \mathrm{KBr}): 1585(\mathrm{w}, \mathrm{N}=\mathrm{N})$,

$1540 \& 1400(\mathrm{~m}, \mathrm{NO} 2), 1140(\mathrm{~s}, \mathrm{~S}=\mathrm{O})$

From the filtrate the solvent was removed and the disulfide (VIII) present in the mixture was solubilized in water as the corresponding mercaptide $(\mathrm{V}, \mathrm{SBr}=\mathrm{SNa})$ by boiling with ethanolic alkaline solution of sodium sulfide (300mg of $\mathrm{NaOH}$ in $50 \mathrm{ml}$ of water $+50 \mathrm{ml}$ of ethanol).

The diphenyl thioether (IX) remained insoluble and was filtered off, washed with water and dried. Yield:120mg 
Crystallized from light petrol (bp 60-80) as red plates. Mp: 243-45

\section{Analysis: C24H16N6O4S}

Requires: C, 59.5; $\mathrm{H}, 3.3$ and N, $17.4 \mathrm{pc}$

Found: C, 59.2; H, 3.6 and N, $17.2 \mathrm{pc}$

\section{$\underline{\text { UV \& visible spectra: }}$}

$\lambda$ max (nm, benzene) $420\left(\varepsilon \dot{m a x} 950, \mathrm{n} \rightarrow \pi^{*}\right), 355\left(\varepsilon \dot{m a x} 11000, \pi \rightarrow \pi^{*}\right)$

\section{$\underline{\text { IR spectra: }}$}

$v(/ \mathrm{cm}, \mathrm{KBr}) 1585(\mathrm{w}, \mathrm{N}=\mathrm{N}), 1540 \& 1400(\mathrm{~m}, \mathrm{NO} 2)$

The filtrate containing sodium mercaptide $(\mathrm{V}, \mathrm{SBr}=\mathrm{SNa})$ was acidified with moderately strong $\mathrm{HCl}$ and the resulting mixture was heated in a beaker for 5 minutes and then was allowed to stand at room temperature for 1 hour. The mercaptan $(\mathrm{V}, \mathrm{SBR}=\mathrm{SH})$ formed on acidification underwent oxidation to the disulfide (VIII), which was then filtered off, washed and dried.

\section{Yield: $150 \mathrm{mg}$}

Crystallized from benzene-ethanol mixture (90:10 v/v) as red crystals. Mp: 197-98

\section{Analysis: C24H16N6O4S2}

Requires: C, 55.8; $\mathrm{H}, 3.1$ and $\mathrm{N}, 16.3 \mathrm{pc}$

Found: C, 55.5; H, 3.7 and N, $16.1 \mathrm{pc}$

\section{UV and visible spectra:}

$\lambda \operatorname{maxi}\left(\mathrm{nm}\right.$, benzene): 425 (غ́max 900, $\left.\mathrm{n} \rightarrow \pi^{*}\right), 360\left(\dot{m} \max 12000, \pi \rightarrow \pi^{*}\right)$

\section{IR spectra:}

v (/cm, KBr) $1590(\mathrm{w}, \mathrm{N}=\mathrm{N}), 1540$ and $1395(\mathrm{~m}, \mathrm{NO} 2)$

\section{REACTION OF SULFENYL BROMIDE (v) WITH 3 MOLAR PROPORTION OF AQUEOUS SODIUM HYDROXIDE}

To an aq solution of sulfenyl bromide $(1.068 \mathrm{gm}, \sim 0.003 \mathrm{~m})$ was added an aq solution of sodium hydroxide ( 6 $\mathrm{ml}$ of $6 \%$ solution, $\sim 0.009 \mathrm{~m}$ ) when a red ppt was formed quickly from a transient blue-violet solution. The precipitated disulfide (VIII) was filtered off, washed and dried.

Yield: $400 \mathrm{mg}$ ( $80 \%$ of the theory)

Crystallization from benzene-alcohol mixture (90:10, v/v) as red crystals. Mp \& mixed mp 193-95.

The filtrate on acidification with moderately strong $\mathrm{HCl}$ gave a rose red ppt of the sulfinic acid (XIX), which was filtered off, washed with water and dried.

Yield: $180 \mathrm{mg}(\sim 60 \%$ of the theory)

Crystallized from benzene-light petrol mixture as nearly colourless plates due to the tautomeric form (XX) of the acid. Mp 144-45.

\section{Analysis: C12H9N3O4S}

Requires: C, 49.5; H, 3.1 and N, 14.4 pc

Found: C, 49.4; H, 3.0 and N, 14.0 pc.

\section{UV and visible spectra:}


$\lambda \max \left(\mathrm{nm}\right.$, benzene) $356\left(\dot{\varepsilon} \max , 12500 \pi \rightarrow \pi^{*}\right)$

\section{IR spectra:}

v (/cm, KBr) 3200(b, OH), 1540 and 1390 (m, NO2), 1100 (s, S=O).

\section{Reference:}

[1] Nakamura N, J. Am. Chem. Soc. (105), 7172 (1983)

[2] Chou T. S., Burgtorf N., Ellis A. L., Lammert S. R. and Kukoja S. P., J. Am. Chem. Soc. 96, 1099 (1974)

[3] Hogg D. R. and Vipond P. W., J. Chem. Soc. (13) 1242, (1974)

[4] Hukel A. and Pfiederer W., Tetrahedron Lett. (24), 5047 (1983)

[5] Unpublished work carried out in the Deptt of Chemistry, Gauhati University (1993-94) 